\section{A response to the two faces of autophagy in DCIS}

\author{
Virginia Espina and Lance A. Liotta
}

Regarding our recent Opinion article (What is the malignant nature of human ductal carcinoma in situ? Nature Rev. Cancer 11, 68-75 (2011)) $)^{1}$ we thank Drs Yu and Shao for their correspondence (The two faces of autophagy and the pathological underestimation of DCIS. Nature Rev. Cancer XX July 2011 (doi:10.1038/nrc2950-c1)) $)^{2}$, which provides interesting discussion points relative to our proposals on autophagy therapy for ductal carcinoma in situ (DCIS) $)^{1,3}$. Drs $\mathrm{Yu}$ and Shao raise three points: that although anti-autophagy therapy can target a survival mechanism used within the hypoxic and nutrient-deprived intraductal microenvironment of DCIS, anti-autophagy therapy may not be desirable for treating invasive breast cancer; that because of the sampling error inherent in core-needle biopsies, patients may be diagnosed with DCIS who have invasive cancer that was missed by the biopsy; and whether chloroquine phosphate can penetrate into the nonvascular interior of the DCIS lesion that is surrounded by the basement membrane.

With regard to the first point, autophagy is an evolutionarily conserved and tightly regulated mechanism that targets intracytoplasmic components to lysosomes, thereby providing a source of energy during nutrient starvation or stress ${ }^{4,5}$. Autophagy has been shown to provide a means for breast cancer cells to avoid apoptosis and survive in the face of trastuzimab or tamoxifen therapy ${ }^{6,7}$. Beyond breast cancer treatment, chloroquine therapy has also been shown to reduce lymphoma progression ${ }^{8,9}$ and to arrest pancreatic cancer in animal models ${ }^{10,11}$, and it has also been used in clinical trials of glioblastoma ${ }^{12}$. Thus, it is premature to assume that the short-term anti-autophagy therapy, proposed in our preventing invasive neoplasia with chloroquine (PINC) trial (clinical trial number: NCT01023477) to target DCIS within the intraductal niche, may be detrimental to patients with invasive breast cancer. It can equally be argued, based on published studies, that chloroquine could sensitize invasive cancer to therapies for which autophagy is a means for the tumour cells to survive in the face of therapy ${ }^{6,7,10,11}$.

With regard to the second point raised by the authors, the sampling error in core-needle diagnosis of suspicious breast lesions is an important clinical topic that transcends DCIS diagnosis and treatment. In our PINC trial, all patients will undergo standard-of-care surgical excision and pathological examination of the entire volume of the lesion to minimize the chance that invasive cancer will be missed by a core-needle biopsy.

The third point raised by Drs Yu and Shao, penetration of chloroquine into DCIS lesions, is an important pharmacodynamic consideration for any therapy, whether the agent is a small molecule, antibody or protein. Chloroquine is a small molecule that has been shown to rapidly leave the vascular space to penetrate tissues, enter the lysosomal compartment of cells and penetrate the blood-brain barrier ${ }^{12,13}$. We think that a small molecule such as chloroquine is actually preferable for DCIS therapy because the diffusion of an antibody or other large-molecule inhibitor could be retarded by the DCIS basement membrane diffusion barrier ${ }^{14,15}$. However, determining the efficacy of chloroquine treatment for DCIS is a primary goal of our PINC trial.
Virginia Espina and Lance A. Liotta are at the George Mason University, Center for Applied Proteomics and Molecular Medicine, 10900 University Boulevard, Room 353, Bull Run Hall Manassas, VA 20110, USA. Correspondence to L.A.L. e-mail: Iliotta@gmu.edu doi: 10.1038/nrc2950-c2

1. Espina, V. \& Liotta, L. A. What is the malignant nature of human ductal carcinoma in situ? Nature Rev. Cancer 11, 68-75 (2011).

2. Yu, K.-D. \& Shao, Z.-M. The two faces of autophagy and the pathological underestimation of DCIS. Nature Rev. Cancer 22 Jul 2011 (doi:10.1038/ nrc2950-c1)

3. Espina, V. et al. Malignant precursor cells pre-exist in human breast DCIS and require autophagy for survival. PLOS ONE 5, e10240 (2010).

4. Seglen, P. O. \& Gordon, P. B. Effects of lysosomotropic monoamines, diamines, amino alcohols, and other amino compounds on protein degradation and protein synthesis in isolated rat hepatocytes. Mol. Pharmacol. 18, 468-475 (1980).

5. Settembre, C. et al. TFEB links autophagy to lysosomal biogenesis. Science 332, 1429-1433 (2011).

6. Schoenlein, P. V., Periyasamy-Thandavan, S., Samaddar, J. S., Jackson, W. H. \& Barrett, J. T. Autophagy facilitates the progression of ERa-positive breast cancer cells to antiestrogen resistance. Autophagy 5, 400-403 (2009).

7. Vazquez-Martin, A., Oliveras-Ferraros, C. \& Menendez, J. A. Autophagy facilitates the development of breast cancer resistance to the anti-HER2 monoclonal antibody trastuzumab. PLOS ONE 4, e6251 (2009).

8. Amaravadi, R. K. et al. Autophagy inhibition enhances therapy-induced apoptosis in a Mycinduced model of lymphoma. J. Clin. Invest. 117 326-336 (2007)

9. Maclean, K. H., Dorsey, F. C., Cleveland, J. L. \& Kastan, M. B. Targeting lysosomal degradation induces p53-dependent cell death and prevents cancer in mouse models of lymphomagenesis. J. Clin. Invest. 118, 79-88 (2008).

10. Mirzoeva, O. K. et al. Autophagy suppression promotes apoptotic cell death in response to inhibition of the PI3K-mTOR pathway in pancreatic adenocarcinoma. J. Mol. Med. 16 Jun 2011 (doi: 10.1007/s00109-011-0774-y).

11. Yang, S. et al. Pancreatic cancers require autophagy for tumor growth. Genes Dev. 25, 717-729 (2011).

12. Briceno, E., Calderon, A. \& Sotelo, J. Institutional experience with chloroquine as an adjuvant to the therapy for glioblastoma multiforme. Surg. Neurol. 67, 388-391 (2007).

13. Ducharme, J. \& Farinotti, R. Clinical pharmacokinetics and metabolism of chloroquine. Focus on recent advancements. Clin. Pharmacokinet. 31, 257-274 (1996).

14. Rudnick, S. I. \& Adams, G. P. Affinity and avidity in antibody-based tumor targeting. Cancer Biother. Radiopharm. 24, 155-161 (2009).

15. van Osdol, W., Fujimori, K. \& Weinstein, J. N. An analysis of monoclonal antibody distribution in microscopic tumor nodules: consequences of a "binding site barrier". Cancer Res. 51, 4776-4784 (1991).

Competing interests statement

The authors declare no competing financial interests. 